\title{
Impresa sociale e creazione di valore: una tassonomia dei modelli di misurazione dell'impatto sociale sul territorio
}

\author{
Cecilia Grieco* Gennaro IAsevoli ${ }^{* *}$ Laura MichelinI ${ }^{* * *}$
}

\begin{abstract}
Obiettivo del paper: Il tema del rapporto tra impresa e territorio assume particolare rilevanza nell'attuale scenario competitivo caratterizzato da una profonda trasformazione in cui $i$ confini tra settore profit e non profit sono sempre più labili. In questo contesto si sviluppa l'interesse verso il tema delle imprese sociali e della misurazione del valore da esse generato. Il presente contributo propone una tassonomia dei principali modelli di misurazione dell'impatto sociale descrivendone caratteristiche e profili.

Metodologia: A seguito di un'analisi della letteratura sono stati selezionati $n$. 76 modelli di misurazione dell'impatto sociale ed è stata effettuata una cluster analysis gerarchica.

Risultati: La ricerca ha consentito di far emergere le caratteristiche principali dei modelli di misurazione e di individuare quattro diverse categorie di modelli.

Limiti della ricerca Il campione esamina un contesto ampio di modelli, tuttavia le tecniche utilizzate possono offrire margini di discrezionalità al ricercatore e conseguente parzialità nello svolgimento dell'analisi.

Implicazioni pratiche: I risultati emersi e la tassonomia proposta possono rappresentare un riferimento utile alle organizzazioni per orientarsi nell'ampio e variegato panorama dei modelli esistenti. La classificazione è inoltre a vantaggio delle istituzioni pubbliche che, nell'erogazione dei contributi a sostegno delle iniziative sociali, hanno iniziato a tenere conto dei risultati effettivamente ottenuti.

Originalità del lavoro: A fronte di una molteplicità di modelli di misurazione dell'impatto sociale, diversi per caratteristiche e finalità, $i$ sistemi di classificazione sono ancora lacunosi. I criteri identificati in questo contributo sono applicati ad un campione ampio di modelli al fine di pervenire ad una classificazione generale.

Parole chiave: impresa sociale; impatto sul territorio; valore sociale ed economico; impatto sociale; modelli di misurazione

\footnotetext{
Dottorando di Comunicazione, Interculturalità e Organizzazioni Complesse - Università LUMSA

e-mail: c.grieco@lumsa.it

e-mail: iasevoli@lumsa.it

e-mail: 1.michelini@lumsa.it
}

** Ordinario di Economia e Gestione delle Imprese - Università LUMSA

*** Docente a contratto di Economia e Gestione delle Imprese - Università LUMSA
\end{abstract}


Purpose of the paper: The relationship between business and community is becoming a relevant topic especially considering the competitive scenario characterized by deep changes in which the boundaries between profit and not-for-profit organizations are becoming blurred. So, in this context the literature on social entrepreneurship and the measurement of social impact is increasing. The aim of the paper is to develop a taxonomy of existing social impact measurement models, in order to describe their characteristics and aims.

Methodology: Starting from the literature analysis $n .76$ models were selected and a hierarchical cluster analysis was developed.

Findings: The research allows the description of the main characteristics of social impact measurement models and their classification in four categories.

Research limits: The sample examines a large number of existing models, nevertheless the analysis techniques may provide room for discretion to the researcher.

Practical implications: Emerged results can be a useful landmark for companies to address the landscape of existing models. Moreover, the proposed taxonomy is useful for public institutions, which had begun to assess the real achieved outcomes when funding social initiatives.

Originality of the study: Since models are different in nature and purpose, classification systems are still incomplete. Identified criteria are applied to a wide sample of model in order to achieve a general classification.

Key words: social enterprise; impact on the ecosystem; social and economic value; social impact; measurement models

\section{Introduzione}

Il binomio impresa-territorio è ricco di significati e carico di prospettive di analisi che nel corso degli anni hanno dato vita ad ampi dibattiti scientifici. Un tema che assume una particolare rilevanza nell'attuale scenario competitivo, caratterizzato da un momento di profonda trasformazione che interessa le finalità stesse dell'agire d'impresa e che sta portando a ridefinire i confini tra settore profit e non profit, oggi sempre più labili. Da una parte, infatti, vi sono le imprese profit che guardano alle questioni sociali come una nuova sfida competitiva nella piena consapevolezza del fatto che il rapporto che le lega alla società ed al territorio non è ineluttabilmente caratterizzato da istanze conflittuali e da necessità contrapposte, bensì esiste una relazione di interdipendenza reciproca. Come osservano Porter e Kramer (2006) "le grandi imprese di successo hanno bisogno di una società sana. (...) Allo stesso tempo, una società sana ha bisogno di imprese di successo". E questa dipendenza reciproca diventa oggi terreno fertile sul quale si sfidano le imprese e che conduce verso una prospettiva emergente dalla quale partire per ripensare alle strategie competitive. La competitività delle imprese si gioca, infatti, anche sulla capacità di cogliere istanze e necessità utili ad alimentare il benessere comune seguendo quello che gli stessi autori definiscono "creazione di valore condiviso". Inoltre, una comunità sana è generatrice di una domanda crescente e le imprese che perseguono le proprie finalità a scapito della società in cui operano si renderanno conto che il successo è temporaneo (Porter e Kramer, 2006). 
Dall'altra parte, vi è il terzo settore che ha iniziato a far proprie competenze, strumenti e modelli tipici del mondo profit; da qui nasce e si sviluppa il filone di studi sull'imprenditorialità sociale (o social entrepreneurship) e con esso, come vedremo nel paragrafo successivo, si alimenta il dibattito sui nuovi modelli di business sociali (Fiorentino et al., 2012; Michelini, 2012). Negli ultimi anni, infatti, si sono sviluppate nuove forme di imprese definite come "integrate" o "ibride", ossia imprese che modificano i confini tra il mondo profit e quello non profit (Hockerts, 2006). Il ruolo di queste imprese ha assunto una rilevanza tale da formare il cosiddetto "quarto settore" (Fourth Sector Network, 2009).

Queste nuove forme organizzative vanno ad alimentare il tessuto imprenditoriale nella direzione dell'economia civile, in cui imprese sociali e civili ${ }^{1}$ possono contribuire allo sviluppo di un'economia umanizzata (Zamagni, 2007), anche perché capaci di convivere fianco a fianco con le imprese capitalistiche "fino a rappresentare una condizione necessaria (pur non sufficiente) per consentire al mercato di svolgere appieno il suo ruolo di regolatore dell'economia" (Zamagni, 2007, p. 203).

In tale scenario, il tema della misurazione del valore sociale generato da queste nuove forme di imprese diventa fondamentale soprattutto per le istituzioni pubbliche che da una parte trovano in questi modelli di impresa una risposta alle difficoltà di far fronte alle esigenze sociali, ma dall'altro devono poter sostenere finanziariamente il "quarto" settore avendo una garanzia del valore sociale generato.

In questo scenario si pone come esigenza crescente il problema della misurazione del valore laddove, come è stato ribadito dall'OECD (2010), la valutazione dell'impatto delle innovazioni sociali richiede uno sforzo importante in termini di rendicontazione e identificazione di strumenti di misurazione quantitativi e qualitativi. A questo si aggiunge che le istituzioni pubbliche, nell'erogazione dei contributi a sostegno delle iniziative sociali, hanno iniziato a tener conto non solo degli obiettivi e della validità del progetto ma anche dei risultati effettivamente conseguiti. Ad esempio, si sta diffondendo il cosiddetto Social Impact Bond (Shanmugalingam et al., 2011), un meccanismo di investimenti basato sui risultati sociali ottenuti (social outcome).

A fronte di questa attenzione verso il tema, da parte del mondo istituzionale in primis, si è sviluppata una nutrita letteratura sui modelli di misurazione dell'impatto sociale; tuttavia un sistema condiviso di categorizzazione di tali modelli - capace anche di agevolare le imprese nel processo di identificazione e selezione del modello più appropriato - non è stato ancora individuato, nonostante siano stati già proposti alcuni tentativi di classificazione.

1 In estrema sintesi l'impresa civile agisce sul lato della domanda ed è caratterizzata da un approccio multi-stakeholder; ne è un esempio la cooperativa sociale. Differentemente, l'impresa sociale agisce sul lato dell'offerta con il principale scopo di "umanizzare la produzione" e il modello organizzativo prevalente è quello for profit. Per maggiori approfondimenti si veda Bruni e Zamagni, 2004 pp. 182 e ss. 
A partire da questi presupposti il presente contributo si propone di elaborare una tassonomia dei principali modelli di misurazione dell'impatto sociale delle imprese. Il progetto di ricerca prende avvio da un'analisi della letteratura accademica e manageriale a livello nazionale ed internazionale con particolare riferimento a due filoni di studio: quello dell'impresa sociale e quello della misurazione dell'impatto sociale. Successivamente, è stato selezionato un campione di modelli di misurazione dell'impatto sociale ed è stata condotta una cluster analysis gerarchica per pervenire all'elaborazione della tassonomia. Infine, sono state evidenziate le principali conclusioni e implicazioni manageriali.

\section{Impresa sociale: definizioni e approcci in letteratura}

Quello dell'imprenditorialità sociale (o social entrepreneurship) è un filone di studi relativamente recente; nonostante ciò la letteratura sul tema è piuttosto ampia e allo stesso tempo gli approcci e le definizioni sono spesso in contrasto.

Molteplici autori hanno analizzato le principali definizioni di social entrepreneurship. In particolare, Dacin et al. (2010) nell'analisi della letteratura prodotta sul tema dal 1991 al 2010 individuano 37 differenti definizioni di impresa sociale. L'OECD ne analizza 29, diffuse nel periodo dal 2000 al 2010. Inoltre, Swanson e Zhang (2010) identificano 14 differenti costrutti teorici sviluppati tra il 1998 e il 2010 per l'analisi dell'impresa sociale.

Gli studi citati pongono dunque in evidenza la molteplicità di interpretazioni e la difficoltà tutt'oggi evidente di pervenire ad una definizione condivisa.

Innanzitutto, è utile chiarire la distinzione tra imprenditorialità sociale, imprenditore sociale, impresa sociale e corporate social entrepreneurship.

L'imprenditorialità sociale riguarda generalmente il processo o comportamento mentre la definizione di imprenditore sociale fa riferimento al fondatore di un'impresa sociale. In particolare, gli imprenditori sociali sono individui che portano avanti attività orientate ad una missione sociale mantenendo le caratteristiche di un imprenditore come il dinamismo, il coinvolgimento personale e l'orientamento all'innovazione (UNDP e EMES, 2008). L'impresa sociale rappresenta l'output concreto del processo di imprenditorialità sociale. Quando lo stesso processo è attivato da un'impresa già presente sul mercato si fa riferimento alla corporate social entrepreneurship (CSE), (Austin e Reficco, 2009).

L'interpretazione di impresa sociale varia da una prospettiva più ristretta ad una più allargata (Perrini, 2007).

Nel primo caso, meno diffuso, si considera questo fenomeno come una modalità di gestione di un'organizzazione non profit basata sulla capacità di acquisizione di risorse secondo schemi più tipicamente manageriali (Austin et al., 2003).

Per il United Nation Development Programme e l'European Research Network, l'impresa sociale è un'organizzazione imprenditoriale, privata e autonoma, che fornisce beni e servizi con la finalità specifica di generare un beneficio per la comunità; è gestita da (o di proprietà di) gruppi di cittadini, e gli interessi materiali 
degli investitori privati sono soggetti a limiti. Nelle imprese sociali la distribuzione dei profitti deve essere legalmente vietata o strutturata in modo tale da escluderli come principale obiettivo (UNDP e EMES, 2008).

Un'interpretazione simile è fornita da Yunus $(2008 ; 2010)$ il quale definisce il social business come una sotto categoria della social entrepreneurship che opera come un'impresa, vendendo prodotti e servizi ai clienti. Differentemente da un'impresa tradizionale, i profitti non sono ridistribuiti tra gli shareholder ma vengono reinvestiti nell'impresa stessa. Gli investitori che decidono di contribuire finanziariamente alla costituzione di un'impresa sociale possono rientrare del capitale iniziale investito senza ricevere ulteriori dividendi. Tutto il surplus viene reinvestito per migliorare la qualità dei prodotti o servizi venduti o per ampliare l'impresa ${ }^{2}$. Nel contesto italiano la legge (1.n. 118/05 e d.1. n. 155/06) ha introdotto nell'ordinamento l'impresa sociale mettendo a disposizione degli enti senza scopo di lucro le regole fino ad oggi proprie delle organizzazioni commerciali. Per diventare impresa sociale è necessario rispettare importanti vincoli: il settore di intervento (che deve essere "di particolare rilievo sociale"), l'informazione e il coinvolgimento degli stakeholder, la destinazione degli utili non ai soci ma ad investimento per lo sviluppo dell'impresa, il rendiconto economico e sociale delle attività. A queste condizioni tutte le forme giuridiche possono assumere la veste di imprese sociali (Fiorentini, 2006).

Una prospettiva più estesa tende a considerare l'impresa sociale come un'organizzazione che impiega una formula imprenditoriale per risolvere i problemi sociali (Thompson e Doherty, 2006). In accordo con questa prospettiva vi è la definizione proposta dalla Commissione Europea (2011) che considera l'impresa sociale come "un attore dell'economia sociale il cui principale obiettivo non è generare utili per i suoi proprietari o azionisti, ma esercitare un impatto sociale" (p. $2)^{3}$. Secondo questa prospettiva quindi, la principale caratteristica dell'impresa sociale è il perseguimento di obiettivi sociali e ambientali, indipendentemente dalla forma giuridica e dalla modalità di redistribuzione dei profitti. La definizione, infatti, comprende sia imprese che forniscono servizi sociali e/o beni e servizi destinati ad un pubblico vulnerabile (accesso all'alloggio e alle cure, assistenza a persone anziane o disabili, inclusione, ecc.) sia le imprese inclusive, le cui modalità di produzione di beni o servizi perseguono un obiettivo di natura sociale (es. integrazione sociale e professionale tramite l'accesso al lavoro di persone svantaggiate ma la cui attività può riguardare beni o servizi non di natura sociale), (European Commission, 2011).

2 Yunus $(2008 ; 2010)$ propone anche un modello alternativo di social business; esso fa riferimento a imprese orientate al profitto, possedute e controllate anche da persone economicamente svantaggiate. In questo caso, la finalità sociale risiede nel fatto che gli eventuali dividendi sono distribuiti tra gli "imprenditori sociali" con l'obiettivo di ridurre il loro disagio e rendendo, in tal modo, possibile il superamento della loro condizione.

3 È opportuno precisare che la Commissione Europea utilizza i termini di impresa sociale e social business come sinonimi. 
L'analisi della letteratura mostra inoltre come numerosi studiosi abbiano posto al centro delle loro definizioni il concetto di missione sociale, declinato in forme diverse: affrontare/alleviare significativi problemi e bisogni sociali (Light, 2006), generare un cambiamento sociale (Mair e Marti, 2006), alleviare le sofferenze di uno specifico gruppo di persone (Martin e Osberg, 2007). Tutte le definizioni concordano quindi nel considerare l'imprenditorialità sociale come un mezzo per alleviare i problemi sociali ed incrementare il benessere della collettività. Inoltre, le definizioni pongono in evidenza come la missione sociale sia orientata verso due macro categorie di beneficiari:

- la collettività in generale;

- specifici gruppi di soggetti che vivono una situazione di disagio sociale (poveri, disabili, ecc.).

Volendo proporre una sintesi si possono evidenziare due variabili critiche nella determinazione delle differenti forme di impresa (Dacin et al., 2010; Yunus et al., 2010): la prima riguarda la tipologia di missione, poiché l'impresa sociale si distingue, come è stato evidenziato, per un orientamento sociale. La seconda fa riferimento alla gestione dei profitti distinguendo le organizzazioni non orientate alla generazione di profitti, quelle in cui non sono ammessi dividendi per gli shareholder e quelle che prevedono la redistribuzione degli utili. Utilizzando le due variabili descritte è stata elaborata una matrice (cfr. Fig. 1) che consente di classificare le diverse tipologie di impresa: l'impresa tradizionale, caratterizzata dal perseguimento di una missione fortemente commerciale e dalla redistribuzione degli utili agli azionisti; l'organizzazione non profit in cui vi è assenza di profitti e l'orientamento ad una missione sociale; e l'impresa sociale caratterizzata dal perseguimento di una missione sociale in cui, a seconda delle interpretazioni (allargata o ristretta), è prevista o meno la redistribuzione dei dividendi.

Fig. 1: Imprenditorialità sociale e forme di impresa

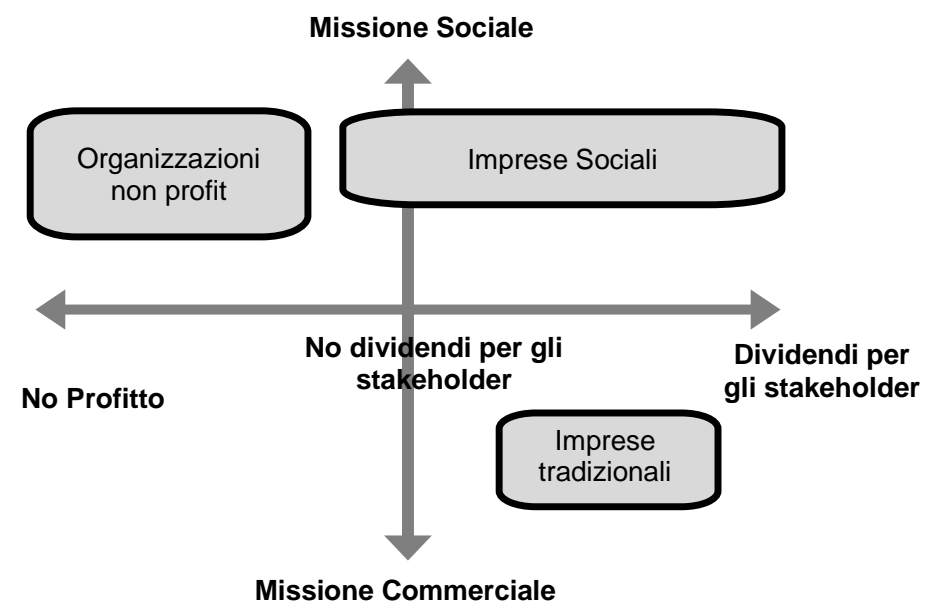

Fonte: ns. elaborazione 
Le definizioni evidenziate dalla letteratura e la classificazione proposta pongono in evidenza l'importanza del ruolo assunto dalla missione sociale come variabile che determina l'essere "sociale" di un'impresa; variabile che funge da discriminante nella classificazione tra imprese tradizionali e sociali. Tuttavia, il concetto di orientamento sociale della mission ha una valenza astratta e teorica se non è integrata ad una prospettiva di misurazione empirica. Da qui la necessità di sviluppare ulteriori riflessioni sui principali modelli utili per la valutazione quantitativa del valore sociale ed economico generato da un'impresa sul territorio di riferimento.

\section{La misurazione dell'impatto sociale generato dalle imprese sociali}

La misurazione delle performance di un'impresa sociale e del loro conseguente impatto in termini non solo economici, ma anche ambientali e sociali è una delle maggiori sfide per la ricerca nell'ambito della social entrepreneurship. Il problema principale non consiste nel processo di misurazione in sé, ma nella traduzione in termini quantitativi dei risultati connessi al perseguimento della causa sociale, prevalentemente di natura qualitativa.

Gli studi esistenti sul tema ruotano attorno al concetto di Blended Value Proposition, delineato da Emerson (2003) per indicare la combinazione di output economici e sociali generati dalle attività di un'organizzazione; secondo questa logica le due tipologie di risultati sono intrinsecamente connesse e richiedono modalità di misurazione olistiche che valorizzino l'entità di entrambe.

Da un'analisi della letteratura a livello nazionale ed internazionale emerge un approccio non univoco al concetto di impatto sociale e di misurazione dello stesso (Maas e Liket, 2010; Nicholls, 2007).

Il social impact viene definito come una combinazione di risorse, input, processi o politiche (Emerson et al., 2000), generati dalla presenza e dall'azione di determinati soggetti (Latanè, 1981) che, nel perseguimento dei risultati desiderati (Reisman e Giennap, 2004), alterano le condizioni di vita, di lavoro, di relazione della popolazione così come le norme ed i valori che orientano la loro cognizione di sé e della società circostante (Burdge e Vanclay, 1996). Il concetto di "impatto" include effetti desiderati e indesiderati, negativi e positivi, che si manifestano sia nel breve che nel lungo periodo (Wainwright, 2002) ed è dato dalla differenza tra gli outcome generati dalle attività di un'organizzazione e ciò che sarebbe successo indipendentemente dal suo intervento (Clark et al., 2004).

Secondo la nostra prospettiva, per impatto sociale si deve intendere la generazione diretta e/o indiretta di un cambiamento, positivo o negativo, prodotto sulla società, sul territorio e sull'ambiente circostante, che coinvolge, quindi, non solo i destinatari diretti, ma tutti coloro che in qualsiasi misura sono coinvolti dall'agire dell'organizzazione.

In letteratura la misurazione del social impact è definita come il processo mediante il quale l'impresa provvede alla rilevazione della propria area di impatto e 
dei gruppi sociali coinvolti (Freudenburg, 1986) ponendo in evidenza la creazione di benefici reali e tangibili che l'operato dell'organizzazione sta apportando; è un processo che rientra in un ambito di ricerca che coniuga pratiche economiche e preoccupazioni sociali e che ne riflette l'interdipendenza (Gentile, 2002).

L'esigenza di delineare sistemi di misurazione idonei non riguarda solo il terzo settore, ma si sviluppa e diffonde in senso più ampio. Nelle imprese for profit, ad esempio, il sorgere di questa tendenza è direttamente proporzionale alla crescente importanza attribuita alle attività di Corporate Social Responsibility, determinando lo sviluppo di indici di misurazione ad hoc. Ad esempio, nel 2012 la The Coca Cola Company ha pubblicato il rapporto "L'impronta socio-economica di Coca Cola in Italia" con l'obiettivo di stimare l'impatto dell'azienda sull'economia italiana in termini di valore aggiunto e generazione dell'occupazione: l'azienda genera un valore aggiunto sull'economia italiana pari a 3.163 milioni di euro ed un impatto occupazionale diretto ed indiretto pari a 45.300 posti di lavoro.

La stessa esigenza emerge anche nel settore pubblico dove, però, gli sforzi effettuati rimangono confinati al settore sanitario e, in misura minore, a quello assistenziale (Zappalà e Lyons, 2009).

Lo sviluppo di modelli di misurazione non è un'attività recente per le imprese sociali, né più in generale per il terzo settore. L'elemento di differenziazione rispetto al passato deriva dal contesto competitivo in cui queste imprese si confrontano oggi, il quale comporta la necessità di valutare puntualmente l'impatto per migliorare le performance e comunicarle in maniera efficace.

Il tentativo di allargarsi ad una prospettiva differente rispetto a quella economica tradizionale, si scontra, però, con la più radicata convinzione circa l'estrema difficoltà se non addirittura l'impossibilità di misurare e quantificare la creazione di valore sociale da parte dell'impresa (Emerson et al., 2000). Infatti, sebbene esistano principi di contabilità generalmente accettati per guidare la rendicontazione finanziaria, non esiste ancora uno standard comparabile relativo alla misurazione e comunicazione dell'impatto sociale: data la difficile identificazione univoca del concetto, gli strumenti per misurarne l'entità sono talvolta privi del rigore che invece contraddistingue approcci più scientifici e sofisticati con i quali si misurano i ritorni economici (Zappalà e Lyons, 2009).

Nonostante vi siano innegabili difficoltà, una misurazione efficiente presenta numerose opportunità strategiche per gli imprenditori sociali (Nicholls, 2005). In primo luogo è un processo che può migliorare le performance dell'impresa poiché consente una comprensione più approfondita circa le modalità di allocazione delle risorse interne al fine di massimizzare il risultato sociale; consente, inoltre, di migliorare le procedure di rendicontazione e permette all'impresa di costruire la sua legittimità di operare, di ottenere e mantenere risorse all'interno della propria rete di stakeholder (Dart, 2004).

Le motivazioni che spingono le imprese sociali ad avviare un processo di misurazione dell'impatto sociale generato possono essere differenti, ma principalmente l'esigenza di relazionarsi con gli stakeholder, è un fattore determinante di importanza considerevole. Le imprese sociali, infatti, possono 
utilizzare la quantificazione del loro impatto sociale come informazione ulteriore al fine di dimostrare il valore aggiunto creato ed i benefici che il loro operato apporta alla società. Trattandosi di un dato relativo al modo in cui gli investimenti ricevuti vengono utilizzati per affrontare determinati problemi sociali, tale informazione risulta strategica da comunicare, soprattutto in un contesto in cui il fundraising è sempre più competitivo. A tale proposito è rilevante l'interesse dei soggetti che investono in queste imprese con la finalità principale di sostenere la causa sociale promossa; per questi ultimi l'esistenza di pratiche condivise relative alla misurazione ed alla comunicazione realistica ed effettiva del raggiungimento di un obiettivo sociale, è fondamentale al fine di poter valutare in modo ottimale i progetti da sostenere (Ruttman, 2012).

Nei contributi esistenti in materia sono identificabili due principali ambiti di approfondimento. Da un lato, gli studi che si focalizzano sullo sviluppo e l'analisi di modelli ed indicatori volti a misurare le performance in modo efficace; dall'altro, più rare, vi sono le ricerche finalizzate a sistematizzare e classificare i modelli esistenti, definire un vocabolario comune e sviluppare un set di criteri per la valutazione dei metodi (Clark et al., 2004, Maas e Liket, 2010; Nicholls, 2005; Zappalà e Lyons, 2009; Rinaldo, 2010). L'obiettivo di questi contributi è delineare un sistema universale, che consenta alle organizzazioni di misurare e comunicare il proprio impatto sociale seguendo un framework di riferimento comune ed univoco nella scelta del metodo più idoneo alle proprie esigenze. Ciò contribuirebbe ad incrementare l'affidabilità e la trasparenza delle rendicontazioni, consentendo al tempo stesso una comparazione significativa delle performance tra organizzazioni diverse all'interno di un settore o di un'area territoriale.

Il processo di misurazione si fonda sul concetto di Impact Value Chain, che permette di differenziare gli output dagli outcome, e facilita il cambiamento di prospettiva dai primi ai secondi, necessario per identificare il contributo effettivo delle attività dell'organizzazione nella realizzazione di un cambiamento sociale.

Fig. 2: Impact Value Chain

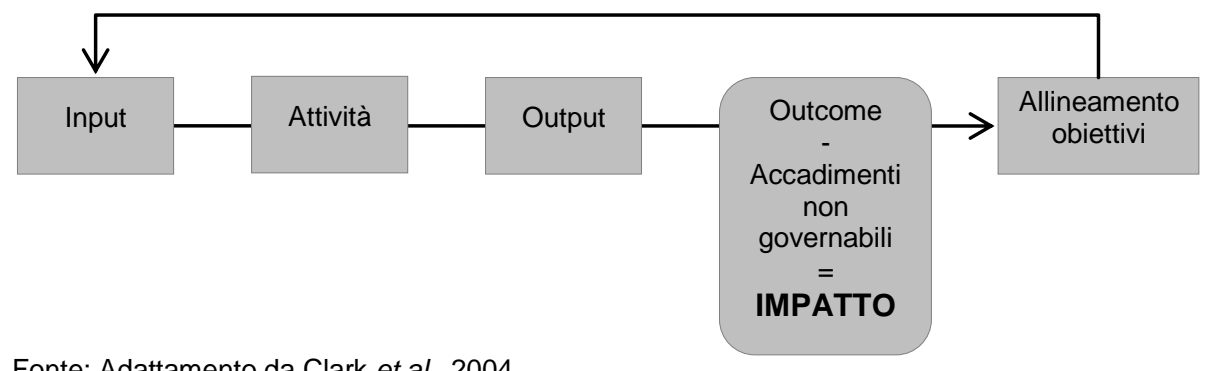

Fonte: Adattamento da Clark et al., 2004

Gli output sono i risultati tangibili e direttamente imputabili all'attività dell'organizzazione in questione, gli outcome, invece, sono i cambiamenti che occorrono nella realtà circostante e che l'organizzazione contribuisce a creare. 
Un'impresa deve pertanto definire gli outcome desiderati e identificare gli output tangibili che possono contribuire a realizzarli nel lungo periodo.

\section{La classificazione dei modelli di misurazione dell'impatto sociale}

I contributi sul tema della misurazione dell'impatto sociale che si focalizzano sulla classificazione dei modelli esistenti non sono numerosi e identificano criteri di categorizzazione parzialmente convergenti.

Da un'analisi della letteratura sono emersi sette principali sistemi di classificazione di cui alcuni presentano una struttura meno articolata, altri più complessa. Nicholls (2005), ad esempio, classifica i modelli in base alla natura qualitativa o quantitativa dei dati richiesti, mentre Zappalà e Lyons (2009) si focalizzano sulle caratteristiche del modello, distinguendo i "metodi" che definiscono indicatori specifici, dai "framework" che invece forniscono linee guida più generali.

Altri autori propongono invece set di criteri più complessi (cfr. Tab. 1), ad esempio, Maas e Liket (2010) identificano 6 variabili e 17 sottocategorie di analisi.

Tab. 1: Le variabili di classificazione dei modelli di misurazione dell'impatto sociale in letteratura

\begin{tabular}{|c|c|c|}
\hline AUTORE & ANNO & VARIABILI DI CLASSIFICAZIONE \\
\hline TRASI & 2012 & $\begin{array}{ll}\text { - } & \text { Scopo (verifica, gestione, certificazione) } \\
\text { - } & \text { Tipologia di organizzazione (non profit, governi, fondazioni, imprese } \\
& \text { sociali, investitori sociali) } \\
\text { - } & \text { Settore (generale, specifico) } \\
\text { - } & \text { Oggetto (efficienza, impatto sociale) } \\
\text { - } & \text { Fase della Impact Value Chain (output, outcome, impact) }\end{array}$ \\
\hline \begin{tabular}{|l} 
New Economy \\
Foundation (NEF)
\end{tabular} & 2012 & $\begin{array}{ll}- & \text { Dimensione dell'impresa (grande, media, piccola) } \\
\text { - } & \text { Oggetto della valutazione (strategia, qualità, impatto) } \\
\text { - } & \text { Tipologia di impatto (ambientale, economico, olistico, sugli individui) }\end{array}$ \\
\hline Rinaldo & 2010 & $\begin{array}{l}\text { - Motivazione della misurazione (valutazione efficienza e/o efficacia, } \\
\text { attuazione di un cambiamento, certificazione, richiesta finanziatori) } \\
\text { - } \quad \text { Preparazione al processo (obiettivo sociale definito, non definito) } \\
\text { - } \quad \text { Risorse disponibili (poche, medie, molte) } \\
\text { - } \quad \text { Tipologia di impatto (ambientale, economico, olistico, sui volontari) }\end{array}$ \\
\hline Maas e Liket & 2010 & $\begin{array}{ll}\text { - } & \text { Scopo (controllo, monitoraggio, comunicazione, valutazione) } \\
\text { - } & \text { Prospettiva temporale (antecedente, concomitante, retrospettiva) } \\
\text { - } & \text { Oggetto della misurazione (input, output ) } \\
\text { - } & \text { Periodo di riferimento (breve termine, lungo termine) } \\
\text { - } & \text { Prospettiva dimensionale (micro, meso, macro) } \\
\text { - } & \text { Approccio (processo, misurazione, monetizzazione) }\end{array}$ \\
\hline Zappalà e Lyons & 2009 & - Metodi e framework \\
\hline Nicholls & 2005 & - Qualitative e quantitative \\
\hline Clark et al. & 2004 & $\begin{array}{ll}\text { - } & \text { Funzione (processo, misurazione, monetizzazione) } \\
\text { - } & \text { Fase Impact Value Chain (input, attività, output, outcome, obiettivi } \\
& \text { raggiunti) } \\
\text { - } & \text { Fase del ciclo di vita dell'organizzazione (avvio, espansione, maturità) } \\
\text { - } & \text { Scopo (controllo, creazione di partnership, gestione, misurazione, } \\
& \text { comunicazione esterna, valutazione retrospettiva) } \\
\text { - } & \text { Rapporto tempo/costi } \\
\text { - } & \text { Time breakdown (manager, staff, consulenti di terza parte, investitori) }\end{array}$ \\
\hline
\end{tabular}

Fonte: ns. elaborazione 
I contributi esaminati, nonostante propongano criteri di classificazione esaustivi, presentano alcune lacune. In particolare, Maas e Liket (2010), Zappalà e Lyons (2009) e Nicholls (2005) si limitano ad un'analisi puramente descrittiva delle variabili e dei criteri di classificazione o svolgono un'analisi quantitativa su un numero ridotto di modelli; altri (Clark et al., 2004; Rinaldo, 2010) li analizzano come casi di studio; infine, TRASI e NEF utilizzano le variabili ai fini della costruzione di un database per l'archiviazione dei dati.

A partire da questi presupposti il presente lavoro si propone di colmare le lacune evidenziate dallo studio della letteratura attraverso la realizzazione di un'analisi quantitativa dei modelli di misurazione esistenti al fine di pervenire ad una classificazione generale.

\section{Metodologia della ricerca}

Obiettivo del presente lavoro è quello di realizzare una tassonomia dei modelli di misurazione dell'impatto sociale esistenti in letteratura attraverso l'elaborazione di una cluster analysis gerarchica.

Per la selezione del campione sono state svolte le seguenti attività:

1. ricerca di articoli accademici su due banche dati internazionali (EBSCO e Google Scholar) contenenti nel titolo o nell'abstract le seguenti parole chiave: "social impact and measurement", "social impact and assessment" e "social impact and tool". La ricerca è stata effettuata nel mese di Aprile 2012. Dalla ricerca sono emersi complessivamente 1215 articoli;

2. selezione, attraverso la lettura degli abstract e del full text, degli articoli rilevanti. Sono stati selezionati 139 articoli;

3. selezione dei modelli citati o descritti negli articoli selezionati (27);

4. integrazione dei modelli individuati attraverso la ricerca su due banche dati di rilievo internazionale: il Foundation Center (TRASI) ${ }^{4}$ e la New Economics Foundation $(\mathrm{NEF})^{5}$. La ricerca è stata effettuata nel mese di Maggio 2012. Dalla ricerca sono emersi complessivamente $173+21$ modelli;

5. selezione di modelli di misurazione dell'impatto sociale $(42+20)$;

6. individuazione di tutti $\mathrm{i}$ modelli di misurazione dell'impatto sociale ed eliminazione dei duplicati (13), (cfr. Appendice 1).

4 Il Foundation Center è un'associazione che raccoglie 550 Fondazioni che operano in tutto il mondo. Nel 2010 ha lanciato il progetto TRASI (Tools and Resources for Assessing Social Impact) un database contenente più di 150 modelli per misurare l'impatto di programmi ed investimenti sociali, fornendo informazioni sulle strategie implementate per la misurazione e la valutazione. Gestisce inoltre un portale dedicato ad organizzazioni non profit, finanziatori ed imprese sociali per lo scambio e la condivisione delle esperienze.

5 La New Economics Foundation (NEF) è un'organizzazione non profit che svolge, a livello internazionale, attività di ricerca sulle tematiche legate all'economia sociale e ambientale. Lavora in partnership con il New Economics Insitute degli Stati Uniti. 
I modelli sono stati selezionati (fasi 3 e 5) escludendo quelli non coerenti con le finalità della ricerca. Nello specifico sono stati esclusi: - i modelli focalizzati esclusivamente sulla valutazione dell'efficienza interna dell'impresa; - i modelli non direttamente riferiti alle imprese (come ad esempio quelli che analizzano l'impatto delle attività governative); - i modelli per cui le informazioni disponibili non erano sufficienti all'analisi.

Fig. 3: Il processo di selezione del campione

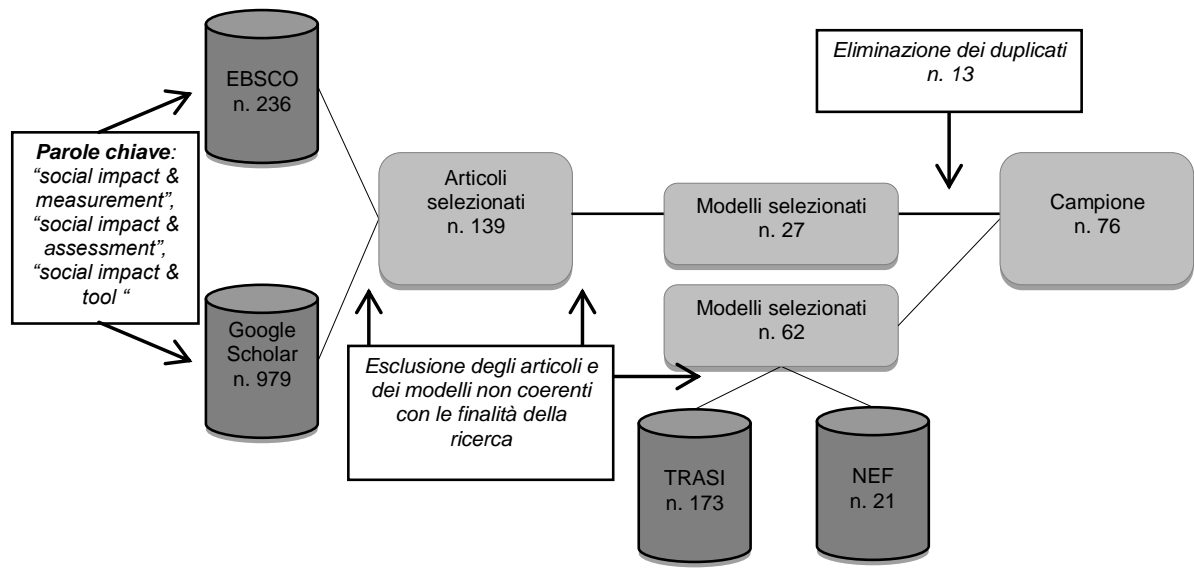

Fonte: ns. elaborazione

Nella Figura 3 sono evidenziate le fasi di raccolta e selezione dei modelli e i relativi output quantitativi.

Dall'integrazione delle banche dati sono stati individuati n. 76 modelli di misurazione dell'impatto sociale che rappresentano il campione oggetto dell'analisi.

Per la cluster analysis le variabili di classificazione sono state selezionate sulla base dell'analisi della letteratura (cfr. Par. 4) tenendo conto dei seguenti criteri: frequenza con cui le variabili sono state utilizzate e possibilità di accesso alle informazioni necessarie.

Nello specifico sono state selezionate le seguenti variabili ${ }^{6}$ :

1. tipologia dati in input: quantitativi, qualitativi, quali-quantitativi;

2. oggetto prevalente dell'analisi: olistico (basato su un'analisi complessiva del valore aggiunto generato), interno (si focalizza sugli aspetti interni, es. volontari, clima organizzativo), ambientale, sociale (sono presenti indicatori di misurazione strettamente connessi all'impatto sociale, es. inclusione), economico;

3. scopo: verifica (quando il modello si propone di verificare il raggiungimento di obiettivi specifici), misurazione (si focalizza sulla quantificazione dell'impatto

6 Per consentire il raggruppamento per cluster ciascuna sottocategoria è stata trattata come variabile binaria $(0 / 1)$. 
sociale generato), gestione (è impiegato come strumento di supporto e monitoraggio nello svolgimento delle attività), certificazione (volto all'ottenimento di una certificazione), reporting (si propone di generare una reportistica per la rendicontazione agli stakeholder);

4. complessità del modello: basic, semplice, medio e complesso ${ }^{7}$;

5. settore: generale (applicabile a tutti i settori), specifico (rivolto ad imprese che operano in un settore specifico);

6. timeframe: antecedente (allo svolgimento delle attività), concomitante, retrospettivo;

7. soggetto promotore: centro di ricerca/università, non profit network, organizzazione non profit, società di consulenza, istituzione.

È stata quindi elaborata una cluster analysis gerarchica; per la formazione dei cluster è stato utilizzato il metodo di Ward e per determinare la similarità tra soggetti è stata utilizzata la distanza Euclidea. I dati sono stati elaborati con il software IBM Statistics SPSS 20.

\section{Analisi dei risultati}

\subsection{Analisi del campione: principali caratteristiche dei modelli}

Al fine di avere una visione d'insieme circa le caratteristiche principali dei modelli esaminati, è stata svolta un'analisi delle frequenze relativamente ai dati osservati per ciascuna variabile (cfr. Tab. 2). In merito all'oggetto prevalente di analisi, i modelli propongono prevalentemente una misurazione olistica $(47,4 \%)$ e sociale $(25 \%)$, mentre sono residuali i modelli che hanno come oggetto l'analisi dell'impatto economico ed ambientale (entrambi pari a 5,3\%). Lo scopo principale risulta essere la verifica dell'operato $(44,6 \%)$. Con riferimento alla complessità la maggior parte del campione risulta costituito da modelli basic $(40,8 \%)$ e semplici $(27,6 \%)$, ossia con un numero di indicatori inferiori a 40 . Solo il $6,6 \%$ è costituito da modelli complessi con più di 90 indicatori. L'analisi mostra una maggioranza di modelli generalisti $(78,9 \%)$, con un orientamento di analisi concomitante e retrospettiva $(96,1 \%)$, ed un utilizzo di dati di natura prevalentemente qualitativa $(47,4 \%)$. Un'ultima osservazione concerne la tipologia dei soggetti promotori, per la maggior parte network di organizzazioni non profit $(31,6 \%)$ e organizzazioni non profit stesse $(30,2 \%)$.

\subsection{Cluster analysis gerarchica: le tipologie di modelli}

Per la determinazione del numero dei cluster è stato costruito il dendrogramma con la gerarchia delle soluzioni (cfr. Appendice 2). È stata scelta la soluzione a

7 Per l'analisi della complessità sono stati calcolati il numero di categorie e indicatori presenti nel modello. 
quattro cluster sulla base dei criteri di numerosità ed interpretabilità dei cluster (valori di significatività per ciascuna variabile impiegata). Sono state quindi elaborate le tabelle di contingenza che incrociano le variabili utilizzate per la classificazione con i cluster formati. Nella Tabella 3 sono sintetizzati i valori del Chi-quadrato di Pearson che emergono per ciascun incrocio ed i livelli di significatività. Come si può osservare tutte le variabili generano differenze significative nei cluster (sig. $<0,05$ ).

Tab. 2: Analisi delle frequenze

\begin{tabular}{|lr|}
\hline VARIABILE & FREQUENZA \\
\hline Oggetto prevalente di analisi & \\
\hline Olistico & $47,4 \%$ \\
\hline Persone & $17,1 \%$ \\
\hline Sociale & $25 \%$ \\
\hline Ambientale & $5,3 \%$ \\
\hline Economico & $5,3 \%$ \\
\hline Scopo & \\
\hline Verifica & $44,6 \%$ \\
\hline Misurazione & $13,2 \%$ \\
\hline Gestione & $17,1 \%$ \\
\hline Certificazione & $7,9 \%$ \\
\hline Reporting & $17,1 \%$ \\
\hline Complessità & \\
\hline Basic & $40,8 \%$ \\
\hline Semplice & $27,6 \%$ \\
\hline Medio & $25 \%$ \\
\hline Complesso & $6,6 \%$ \\
\hline
\end{tabular}

\begin{tabular}{|lr|}
\hline VARIABILE & FREQUENZA \\
\hline Settore & $78,9 \%$ \\
\hline Generico & $21,1 \%$ \\
\hline Specifico & \\
\hline Timeframe & $3,9 \%$ \\
\hline Antecedente & $40,8 \%$ \\
\hline Concomitante & $55,3 \%$ \\
\hline Retrospettivo & \\
\hline Tipologia di promotore & $19,7 \%$ \\
\hline Università/centro di ricerca & $31,6 \%$ \\
\hline Non profit network & $30,2 \%$ \\
\hline Organizzazione non profit \\
\hline Società di consulenza & $13,2 \%$ \\
\hline Istituzione & $5,3 \%$ \\
\hline Tipologia dati & $47,4 \%$ \\
\hline Qualitativi & $17,1 \%$ \\
\hline Quantitativi & $35,5 \%$ \\
\hline Entrambi
\end{tabular}

Fonte: ns. elaborazione

Tab. 3: Chi-quadrato di Pearson per variabile

\begin{tabular}{|lrrr|}
\hline Chi-quadrato di Pearson & Valore & df & Sig. asint. (2 vie) \\
\hline Tipologia dati & 71,689 & 6 &, 000 \\
\hline Oggetto prevalente di analisi & 31,106 & 12 &, 002 \\
\hline Scopo prevalente & 141,223 & 12 &, 000 \\
\hline Complessità & 33,141 & 9 &, 000 \\
\hline Settore & 10,249 & 3 &, 017 \\
\hline Timeframe & 34,698 & 6 &, 000 \\
\hline Tipologia di promotore & 28,501 & 12 &, 005 \\
\hline
\end{tabular}

Fonte: ns. elaborazione

Da una lettura più approfondita delle caratteristiche dei cluster è stato possibile costruire un profilo descrittivo per ciascun gruppo:

- il cluster 1 (modelli SOCIO-QUANTITATIVI SEMPLICI) rappresenta il 14,5\% del campione ed è formato da modelli basati su indicatori quantitativi che hanno per oggetto prevalente sia il valore sociale generato (es. numero beneficiari dei servizi erogati) sia l'impatto sulle persone (es. soddisfazione del personale, clima organizzativo). Con riferimento al timeframe, i modelli di questo cluster 
misurano l'impatto prevalentemente a posteriori. Tali modelli sono generalmente semplici (costituiti da non più di 15 indicatori) e generalisti, quindi applicabili a qualsiasi settore. Sono proposti da differenti tipologie di soggetti: centri di ricerca/università, network di organizzazioni non profit, organizzazioni non profit, società di consulenza e istituzioni;

- il cluster 2 (modelli OLISTICO-COMPLESSI) rappresenta il 26,3\% del campione ed è formato da modelli caratterizzati da variabili sia qualitative che quantitative. Hanno per oggetto prevalente un'analisi ad ampio spettro (olistica) del valore generato (comprende l'analisi dell'impatto sociale, ambientale ed economico). Gli scopi prevalenti sono la verifica del raggiungimento degli obiettivi e l'attività di reporting. In tal senso, infatti, l'applicazione del modello è spesso volta alla produzione di una documentazione utile per la rendicontazione agli stakeholder. Hanno un grado di complessità elevato (alcuni di essi contengono oltre 100 indicatori) e sono applicabili a qualsiasi settore. Il timeframe prevalente è quello di un'analisi concomitante o retrospettiva e sono proposti prevalentemente da network di organizzazioni non profit;

- il cluster 3 (modelli CONTROLLO-QUALITATIVI) è il cluster più numeroso (40,8\% del campione) ed è caratterizzato da modelli che impiegano variabili qualitative e una prospettiva di analisi olistica. Hanno un timeframe prevalentemente retrospettivo e, anche in virtù della loro natura qualitativa, hanno un livello basic di complessità. I modelli che appartengono a questo cluster possono essere riferiti anche a settori specifici e sono proposti da tipologie di soggetti differenti;

- il cluster 4 (modelli GESTIONALI) rappresenta il 18,4\% del campione, è caratterizzato da modelli che impiegano variabili qualitative o quantitative e l'oggetto dell'analisi può essere di diversa natura (olistico, persone, ambientale, sociale economico). Ciò che caratterizza prevalentemente questo gruppo di modelli è lo scopo: infatti, sono impiegati per l'attività di gestione o certificazione ed applicati durante tutto lo svolgimento delle attività (timeframe concomitante). Sono generalisti e proposti prevalentemente da società di consulenza e istituzioni.

\section{Conclusioni}

L'analisi della letteratura ha posto in evidenza il ruolo crescente delle imprese sociali o cosiddette "ibride" nello scenario economico e nel contesto territoriale in cui esse operano. È emerso, inoltre, come il concetto di social entrepreneurship racchiuda forme di imprese con caratteristiche e modelli di governance differenti ma accomunate dall'orientamento sociale della propria mission. In questo ambito è stata sottolineata da più fonti l'esigenza di stimare in maniera più accurata l'impatto che tali imprese sono in grado di generare per la società e per il territorio di riferimento; in effetti, la misurazione dell'impatto generato, oggi si configura come un importante valore aggiunto per le imprese innanzitutto a fronte dei considerevoli 
vantaggi legati al miglioramento delle performance, ma anche per la possibilità di coinvolgere e rendicontare ai propri stakeholder gli effettivi risultati conseguiti.

L'analisi della letteratura ha posto in evidenza l'esistenza di numerosi modelli di misurazione, con caratteristiche, finalità e modalità di implementazione differenti. Tuttavia, proprio in virtù di una così vasta molteplicità di modelli, si evidenzia una carenza di chiarezza in termini di classificazione.

Lo studio proposto offre innanzitutto una rassegna dei principali modelli di misurazione dell'impatto sociale esistenti a livello mondiale, sviluppati da differenti soggetti: centri di ricerca/università, network di organizzazioni non profit, organizzazioni non profit, società di consulenza e istituzioni.

La cluster analysis svolta ha consentito di individuare quattro diverse categorie di modelli per la misurazione dell'impatto sociale: i modelli socio-quantitativi semplici, i modelli olistico-complessi, i modelli controllo-qualitativi e i modelli gestionali.

I cluster identificati, con la descrizione dei relativi profili caratterizzanti, forniscono un quadro di insieme dei modelli e consentono una visione globale in base alla quale ciascun modello può essere identificato e compreso nei suoi elementi e determinanti principali. I risultati emersi e la tassonomia proposta possono rappresentare un riferimento utile alle organizzazioni per orientarsi nell'ampio e variegato panorama dei modelli esistenti.

Innanzitutto, la singola organizzazione può comprendere le caratteristiche di ciascun modello in termini di oggetto di analisi, obiettivi, complessità, ambito e fase di implementazione. Inoltre, la tassonomia elaborata consente alle imprese sociali di semplificare il processo di selezione del modello più idoneo in base alle caratteristiche distintive ed alle necessità specifiche che si possono manifestare di volta in volta nella gestione delle relazioni con gli stakeholder ed in particolare con gli attori del territorio di riferimento.

D'altra parte, anche gli enti e le istituzioni pubbliche che operano sul territorio hanno iniziato, negli ultimi anni, ad adottare indicatori di misurazione dell'impatto sociale per la selezione dei progetti da sostenere e per la valutazione e il monitoraggio dei progetti finanziati. E l'impiego di questi modelli sarà sempre più importante con la diffusione dei meccanismi di investimento basati sui risultati sociali ottenuti (social outcome). La rassegna teorica e la tassonomia dei modelli proposta possono quindi offrire indicazioni utili anche agli operatori del settore pubblico per l'individuazione dei modelli di misurazione più idonei alle loro necessità.

In conclusione, è opportuno evidenziare che il campione esamina un contesto ampio ma non completo dell'universo dei modelli e inoltre che le tecniche di analisi utilizzate (cluster analysis gerarchica) possono offrire margini di discrezionalità al ricercatore e conseguentemente di parzialità nello svolgimento dell'analisi. 


\section{Appendice 1}

\begin{tabular}{|c|c|c|}
\hline N. & NOME MODELLO & SOGGETTO PROMOTORE \\
\hline 1 & Soft Outcomes Universal Learning & The Research Centre City College \\
\hline 2 & AA1000 & AccountAbility \\
\hline 3 & $\begin{array}{l}\text { Atkisson Compass Assessment for } \\
\text { Investors }\end{array}$ & Atkinsson Group \\
\hline 4 & Best Available Charitable Option & Acumen Fund \\
\hline 5 & BoP Impact Assessment Framework & The William Davidson Institute (WDI) \\
\hline 6 & Co-operative Performance Indicators & Co-operatives UK \\
\hline 7 & Fit for Purpose & Development Trusts Association \\
\hline 8 & Eco-mapping & International Network for Environmen \\
\hline 9 & EMAS & Commissione Europea \\
\hline 10 & $\begin{array}{l}\text { European Foundation for Quality } \\
\text { Management (EFQM) }\end{array}$ & EFQM \\
\hline 11 & $\begin{array}{l}\text { GRI Sustainability Reporting } \\
\text { Framework }\end{array}$ & Global Reporting Initiative \\
\hline 12 & Investors in People & UK Commission for Employment and Skils \\
\hline 13 & Local Multiplier 3 (LM3) & New Economics Foundation (NEF) \\
\hline 14 & Logic Model Builder & Innovation Network \\
\hline 15 & Measuring Impact Framework & WBCSD \\
\hline 16 & Millennium Development Goal Scan & Business in Development and Sustainability \\
\hline 17 & $\begin{array}{l}\text { Practical Quality Assurance System } \\
\text { for Small Organizations }\end{array}$ & Charities Evaluation Services \\
\hline 18 & Prove it! & New Economics Foundation (NEF) \\
\hline 19 & Quality First & Birmingham Voluntary Service Council \\
\hline 20 & $\begin{array}{l}\text { Social IMPact Measurement for Local } \\
\text { Economies (SIMPLE) }\end{array}$ & Social Enterprise London (SEL) and U \\
\hline 21 & SOCIAL & ACCION International \\
\hline 22 & Social Investment Risk Assessment & Hunter Consulting LLC \\
\hline 23 & Social Accounting and Audit & AccountAbility \\
\hline 24 & Social Return on Investment & The SROI Network \\
\hline 25 & The Big Picture & Scottish Council Voluntary Organizations \\
\hline 26 & Third Sector Performance Dashboard & Social Firms UK \\
\hline 27 & $\begin{array}{l}\text { Volunteering Impact Assessment } \\
\text { Toolkit }\end{array}$ & Institute for Volunteering Research \\
\hline 28 & $\begin{array}{l}\text { Impact Reporting and Investment } \\
\text { Standard }\end{array}$ & Global Impact Investing Network \\
\hline 29 & GIIRS & B Lab \\
\hline 30 & $\begin{array}{l}\text { The Values Based Checklist for Social } \\
\text { Firms }\end{array}$ & Social Firms UK \\
\hline 31 & Social Enterprise Balanced Scorecard & Social Enterprise London (SEL) \\
\hline 32 & $\begin{array}{l}\text { Assessment and Improvment } \\
\text { Indicators }\end{array}$ & Venture Philantropy Partners \\
\hline 33 & Charity Analysis Framework & New Philantropy Capital \\
\hline 34 & Cradle to Cradle Certification & $\begin{array}{l}\text { Cradle to Cradle Product Innovation } \\
\text { Institute }\end{array}$ \\
\hline 35 & $\begin{array}{l}\text { Echoing Green Mid-Year and Year } \\
\text { End Report }\end{array}$ & Echoing Green \\
\hline 36 & $\begin{array}{l}\text { HIP (Human Impact + Profit) } \\
\text { Scorecard }\end{array}$ & HIP Investor, Inc. \\
\hline 37 & $\begin{array}{l}\text { Methodology for Impact Analysis and } \\
\text { Assessment }\end{array}$ & Investing for Good \\
\hline
\end{tabular}




\begin{tabular}{|c|c|c|}
\hline 38 & MicroRate & MicroRate \\
\hline 39 & $\begin{array}{l}\text { Movement Above the U.S. \$1 a Day } \\
\text { Threshold }\end{array}$ & Microcredit Summit Campaign \\
\hline 40 & Progress Out of Poverty Index & Grameen Foundation \\
\hline 41 & $\begin{array}{l}\text { Pulse - Portfolio Data Management } \\
\text { System }\end{array}$ & Acumen Fund \\
\hline 42 & SCALERS & Bloom and Chatterji \\
\hline 43 & Expected Return & Hewlett Foundation \\
\hline 44 & Wallace Assessment Tool & Wallace Foundation \\
\hline 45 & Trucost & Trucost PLC \\
\hline 46 & The FINCA Client Assessment Tool & FINCA \\
\hline 47 & The B Impact Rating System & B Lab \\
\hline 48 & TBL Scorecard & Triple Bottom Line Collaborative \\
\hline 49 & Success Measures Data System & NeighborWorks America \\
\hline 50 & Social Value Metrics & Root Capital \\
\hline 51 & Social Rating & Micro Credit Rating International \\
\hline 52 & Social Performance Indicators & CERISE \\
\hline 53 & Acumen Scorecard & Acumen Fund and McKinsey \\
\hline 54 & Cost per impact & $\begin{array}{l}\text { Center for Hight Impacts Philantropy - } \\
\text { University of Pennsylvania }\end{array}$ \\
\hline 55 & $\begin{array}{l}\text { Charity assessment method of } \\
\text { performance }\end{array}$ & Dutch Charities \\
\hline 56 & $\begin{array}{l}\text { Ongoing Assessment of Social } \\
\text { Impacts (OASIS) }\end{array}$ & REDF \\
\hline 57 & Participatory impact assessment & Feinstein International Center \\
\hline 58 & Social Footprint & Center for Sustainable Organizations \\
\hline 59 & $\begin{array}{l}\text { Toolbox for Analysing Sustainable } \\
\text { Ventures in Developing Countrie }\end{array}$ & UNEP \\
\hline 60 & Public Value Scorecard & Hauser Center for Non-profit Corporations \\
\hline 61 & Social Compatibility Analysis & Institute for Sustainable Development \\
\hline 62 & Social Return Assessment & Pacific Community Venture (PCV) \\
\hline 63 & Socio-Economic Assessment Toolbox & Anglo American plc \\
\hline 64 & Stakeholder Value Added & Leuphana Universität Lüneburg \\
\hline 65 & Wellventure Monitor & $\begin{array}{l}\text { Fortis Foudation Netherlands and Erasmus } \\
\text { University Rotterdam }\end{array}$ \\
\hline 66 & Star Social Firm & Social Firms UK \\
\hline 67 & Social Enterprise Mark & Social Enterprise Mark Company \\
\hline 68 & Community Impact Mapping & Development Trust Association \\
\hline 69 & Outcome Star & Triangle Consulting Social Enterprise \\
\hline 70 & Weelbeing Measure & New Philantropy Capital (NPC) \\
\hline 71 & COSA Methodology & Sustainable Commodity Initiative \\
\hline 72 & C3 Perform & Bristol City Council \\
\hline 73 & Family of Measures & Sawhill e Williamson \\
\hline 74 & Customer Service Excellent & EMCQ \\
\hline 75 & Business Ethics Excellence Model & $\begin{array}{l}\text { European Business Ethics Network (EBEN) } \\
\text { - GR }\end{array}$ \\
\hline 76 & SIM Tool Survey & INAFI \\
\hline
\end{tabular}




\section{Appendice 2}

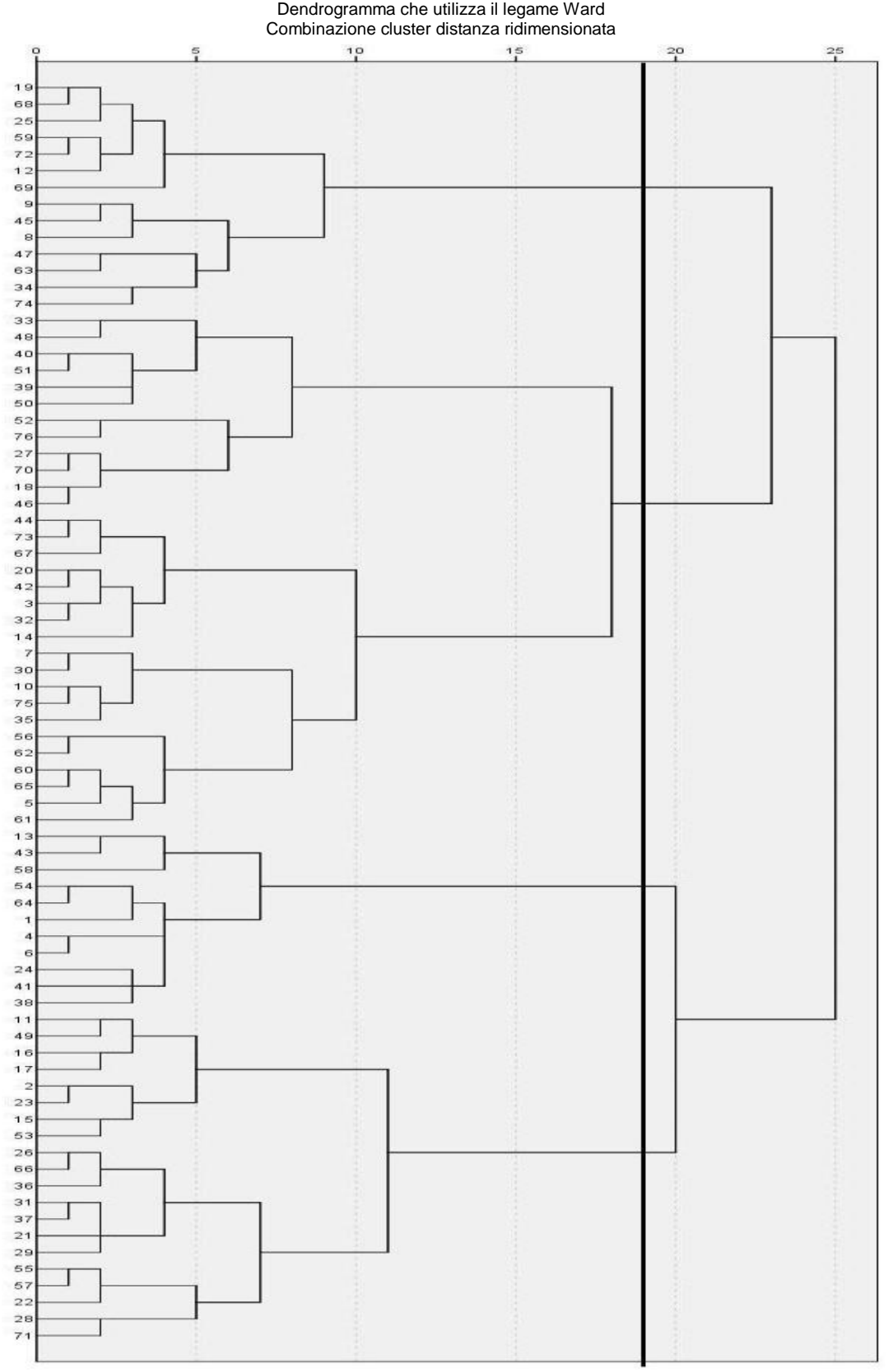




\section{Bibliografia}

AUSTIN J., LEONARD H., REFICCO E., WEI-SKILLEM J. (2005), "Social entrepreneurship: it's for corporations, too", in Nicholls A. (ed), Social Entrepreneurship: New Paradigms of Sustainable Social Change, Oxford University Press, Oxford.

AUSTIN J., REFICCO E. (2009), Corporate social entrepreneurship, Working Paper, n. 09101.

AUSTIN J., STEVENSON H., WEI-SKILLERN J. (2003), "Social entrepreneurship and commercial entrepreneurship: Same, different, or both?", Working paper series, n. 04029, Harvard Business School.

BRUNI L., ZAMAGNI S. (2004), Economia civile: efficienza, equità, felicità pubblica, Il Mulino, Bologna.

BURDGE R., VANCLAY F. (1996), "Social Impact Assessment: A Contribution to the State of the Art Series", Impact Assessment, vol. 14, n. 1, pp. 59-86.

CLARK C., ROSENZWEIG W., LONG D., OLSEN S. (2004), Double bottom line project report: Assessing social impact in double bottom line ventures. Accessibile su http://www.riseproject.org/DBL_Methods_Catalog.pdf. Visitato il 20 Giugno 2012.

DACIN P., DACIN M., MATEAR M. (2010), "Social entrepreneurship: why we don't need a new theory and how we move forward from here", The Academy of Management Perspectives, vol. 24, n. 3, pp. 37-57.

DART R. (2004), "The Legitimacy of Social Enterprise", Nonprofit Management and Leadership, vol. 14, n. 4, pp. 411-424.

EMERSON J. (2003), "The Blended Value Preposition: integrating social and financial return", California Management Review, vol. 45, n. 4, pp. 35-51.

EMERSON J., WACHOWICS J., CHUN S. (2000), Social return on investment: Exploring aspects of value creation in the non-profit sector, The Roberts Foundation, San Francisco.

EUROPEAN COMMISSION (2011), Social Business Initiative. Creating a favourable climate for social enterprises, key stakeholders in the social economy and innovation. Accessibile su http://ec.europa.eu/internal_market/social_business/docs/COM2011 682_en.pdf. Visitato il 15 Maggio 2012.

FIORENTINI G. (2006), Impresa Sociale e sussidiarietà, Franco Angeli, Milano.

FIORENTINO D., IASEVOLI G., MICHELINI L. (2012), "La dimensione territorio nella creazione e nello sviluppo di nuovi modelli di business sociale", in Pilotti L., Creatività, innovazione e territorio, Il Mulino, Bologna.

FOURTH SECTOR NETWORK (2009), The emerging fourth sector. Accessibile su http://www.fourthsector.net/learn/fourth-sector. Visitato il 15 Maggio 2012.

FREUDENBURG WR. (1986), "Social Impact Assessment", Annual Review of Sociology, vol. 12 , pp. 451-478

GENTILE MC. (2002), Social Impact Measuring, a definition, in Business and society program, The Aspen Institute Accessibile su http://www.aspeninstitute.org/sites /default/files/content/docs/bsp/socialimpactmanagement.pdf. Visitato il 15 Maggio 2012.

GIARETTA E. (2005), "Ethical product innovation: in praise of slowness", The TQM Magazine, vol. 17, n. 2, pp.161-181.

HOCKERTS K. (2006), "Entrepreneurial opportunity in social purpose business ventures", in Mair J., Robinson J., Hockerts K., Social Entrepreneurship, Palgrave Macmillan. 
LATANÈ B. (1981), "The psychology of social impact”, American Psychologist, vol. 36, n. 4, pp. 343-356.

LATTIN J., CARROLL D., GREEN P. (2003), Analyzing Multivariate Data, 3rd Edn., Brooks Cole, USA.

LIGHT P.C. (2006), "Reshaping Social Entrepreneurship", Stanford Social Innovation Review, fall, pp.47-51.

MAAS K. (2009), Social Impact Measurement: Towards a guideline for managers. Accessibile su www.erim.eur.nl/portal/page/portal/ERIM/Research/Centres/Erasmus_Centre_for_Str ategic_Philanthropy/Research/Publications/Social_Impact_Measurement_voor_sso_ni euwsbrief\%5B1\%5D.pdf. Visitato il 20 giugno 2012.

MAAS K., LIKET K. (2010), Social Impact Measurement: Classification of Methods. Accessibile su www.erim.eur.nl/portal/pls/portal/xopus_public.download_document? p_guid=9E98A3A73D99B74FE04018AC8A060CB5. Visitato il 20 giugno 2012.

MAIR J., MARTI J. (2006), "Social entrepreneurships research: a source of explanation, prediction and delight", Journal of World Business, vol. 41, n. 1, pp.36-44.

MARTIN R.J., OSBERG S. (2007), "Social entrepreneurship: The case for a definition", Stanford Social Innovation Review, Spring, pp. 29-39.

MICHELINI L. (2012), Social innovation and new business models. Creating shared value in low-income markets, SpringerBriefs in Business, Berlin.

MICHELINI L., FIORENTINO D. (2012), "New business models for creating shared value", Social Responsibility Journal, vol. 8, n. 4. pp. 561-577.

MULGAN G., REEDER N., AYLOTT M., BO'SHER L. (2011), Social Impact Investment: the challenge and opportunity of Social Impact Bonds, The Young Foundation.

NICHOLLS A. (2005), Measuring Impact in Social Entrepreneurship: New Accountabilities to Stakeholders and Investors?, Skoll Centre for Social Entrepreneurship, University of Oxford.

NICHOLLS J. (2007), Why measuring and communicating social value can help social enterprise become more competitive, Cabine Office, Office of the Third Sector. Accessibile su http://evpa.eu.com/wp-content/uploads/2010/09/Why-measuring-andcommunicating-social-value-can-help-social-enterprise-become-morecompetitive1.pdf. Visitato il 10 Maggio 2012.

OECD (2010), SMEs, Entrepreneurship and Innovation. Accessibile su http://www.oecd.org/document/16/0,3746,en_2649_33956792_44938128_1_1_1_1,0 0.html. Visitato il 10 Maggio 2012.

PERRINI F. (2007), Social Entrepreneurship, Egea, Milano.

PORTER M.E., KRAMER M.R. (2006), "Strategy and Society. The link between competitive advantage and corporate social responsibility", Harvard Business Review, December, vol. 84, n. 12, pp. 78-92.

REISMAN J., GIENNAP A. (2004), Theory of change: a practical tool for action, results and learning, Organizational Research Services. Erasmus University Rotterdam. Accessibile su http://www.erim.eur.nl/portal/page/portal/ERIM/.

Research/Centres/Erasmus_Centre_for_Strategic_Philanthropy/Research/Publications /Social_Impact_Measurement_voor_sso_nieuwsbrief\%5B1\%5D.pdf. Visitato il 10 Maggio 2012.

RINALDO H. (2010), Getting started in social impact measurement. The Guild. Accessibile su http://www.gaugeni.co.uk/sites/default/files/Getting\%20started\%20in\%20social \%20impact\%20measurement.pdf. Visitato il 18 giugno 2012. 
RUTTMAN R. (2012), New ways to invest for social and environmental impact, in Investing for impact: how social entrepreneurship is redefining the meaning of return, Credit Suisse Research.

SHANMUGALINGAM C., GRAHAM J., SIMON T., MULGAN G. (2011), Growing social ventures. The role of intermediaries and investors: who they are, what they do, and what they could become. The Young Foundation. Accessibile su http://www.youngfoundation.org/files/images/Growing_Social_Ventures.pdf. Visitato il 15 Maggio 2012.

SWANSON L.A., ZHANG D.D. (2010), “The Social Entrepreneurship Zone”, Journal of Nonprofit \& Public Sector Marketing, vol. 22, n. 2, pp. 71-88.

THOMPSON J., DOHERTY B. (2006), "The diverse world of social enterprise: A collection of social enterprise stories", International Journal of Social Economics, vol. 33 n. 5/6, pp. 361-375.

UNDP, EMES (2008), Social enterprise: a new model for poverty reduction and employment generation. Accessibile su http://europeandcis.undp.org/ourwork/poverty/show/ 02F5569C-F203-1EE9-BD529F7FE21C9320 Visitato il 15 May 2012.

WAINWRIGHT S. (2002), Measuring impact: a guide to resources, NCVO Publications, London.

YUNUS M. (2008), A World Without Poverty: Social Business and the future of capitalism, Public Affairs, New York, USA.

YUNUS M. (2010), Building Social Business, Public Affairs, New York, USA.

YUNUS M., MOINGEON B., LEHMANN-ORTGEGA L. (2010), "Building social business model: lessons from the Grameen experience", Long Range Planning, vol. 43, n. 2/3, pp. 308-325.

ZAMAGNI S. (2007), L'economia del bene comune, Città Nuova, Roma.

ZAPPALÀ G., LYONS M. (2009), Recent approaches to measuring social impact in the third sector: An overview, Centre for Social Impact, Background Paper, n. 5. Accessibile su http://www.csi.edu.au/assets/assetdoc/b20aada17ffad8f7/V2\%20CSI\%20Background $\% 20$ Paper\%20No\%205\%20\%20Approaches\%20to\%20measuring\%20social\%20impa ct.pdf. Visitato il 15 May 2012.

\section{Siti Internet}

TRASI (Foundation Center): http://trasi.foundationcenter.org NEF: http://www.nef-consulting.co.uk 